\title{
LUMBAR DISC PROLAPSE - A FORGOTTEN DIAGNOSIS?
}

\author{
H Stewart, Consultant Orthopaedic Surgeon \\ Royal Lancaster Infirmary
}

\begin{abstract}
We seem to have forgotten what a lumbar disc prolapse is: an increasing number of patients come to the orthopaedic clinics having been inappropriately treated or advised, mostly because the diagnosis has not been made. Appropriate referrals tend to use the phrase 'persistent backache and sciatica', not disc prolapse. It is as if it is not a medically correct term. There is a void in the diagnosis of acute backache which patients want to be filled, and all too often it is filled by paramedical explanations which often leave them confused. This a shame, because it is an easily definable condition, and eminently treatable, the vast majority of patients not needing consultant input at all.
\end{abstract}

There can be few more acutely painful conditions than a full-blown disc prolapse, with the very irritant disc material displacing and irritating a sensitive nerve root. Usually, but not always, the patient feels something 'go' in the lower back when doing an activity involving bending or twisting, and often the pain is slight at first and increases over the next few hours or until the next day. Similarly, it is often the next or subsequent days when the sciatica occurs, as the disc material stirs up intense inflammation. The pain is usually worse with straining and bending, and is generally better when horizontal, although the classic position of lying flat on the back is often painful. Sitting and walking usually aggravate the pain.

The above history is really all that is necessary to diagnose a disc prolapse, indeed most of my diagnoses are made by such a history retrospectively. Examination reveals the other diagnostic indicator (in the presence of sciatica): nerve root irritation signs, or restriction of straight leg raising (SLR). These are not absolutely specific (see Discussion, 5).

The above history also indicates an acute discogenic cause of back pain even if minor, only differing from a full-blown prolapse by intensity or duration, eg disc pain can last as little as a day or even hours. This is important for our advice to patients, as acute disc pathology does NOT improve specifically with activity, it improves fastest with pressure off it (see Discussion, 6). As patients with acute backache will only have spinal imaging when it is severe or persistent, clinical diagnosis is critical, and the history is paramount. Be wary of 'muscle strain' as a diagnosis.

\section{CASE REPORTS}

The following case reports illustrate some further points:

\section{Case 1}

A 38-year-old man presented with a six-month history of sciatica from the buttock to the knee, with no particular causation. He had had some backache six months prior to that, being 'stiff' for a day or so after moving house, and a week later, after pulling a boat onto a beach, he was stiff for two weeks.

His sciatica was acute at first, but at presentation more general and vague. It was made worse by walking, and after 30 minutes or so of driving. It was not made much worse by coughing or sneezing, but was relieved by lying down. His particular grouse at presentation was sciatica after one hundred yards of walking, with back pain going into the buttock and posterior thigh, and pins and needles in his calf and sole of foot.

Initially he had sought physiotherapy privately, which did not include traction, and it had not helped. He then had four sessions of chiropractic treatment to no avail. Then acupuncture, again of no use, but the acupuncturist asked an osteopath to help. At the same time someone told him his legs were unequal in length, and therefore a podiatrist saw him and offered him some 'special shoes or raises', but he demurred. The osteopath said he had a pelvic tilt, and that he could cure this, although it would be 'murder' for a few days. It was, but it did no good. He noticed at this stage that the best thing for him was actually having no treatment, but he did seek specialist help then, and I subsequently saw him.

On examination, his back was straight on standing, but he tilted to the opposite side to his sciatica at the lumbar level on bending forward, and this was restricted, as was extension. He had no weakness or wasting in his legs, his reflexes were present and equal, and he had some blunting of sensation in the right S1 dermatome, ie the outer border of the foot. He had a positive right Bowstring sign (see Discussion, 5) with SLR only 20 degrees.

A diagnosis of a prolapsed disc irritating the right S1 nerve root was made, and he was referred to a physiotherapist. Traction as an outpatient was performed, and over a twoweek period some definite improvement was reported, but to speed things up a caudal epidural of steroid and local anaesthetic was given five weeks after his initial visit. Five weeks later, and after further physiotherapy, he was much improved, and had climbed up Gummers Howe. He was discharged then, but returned five months after original presentation with some sciatica on standing or walking, although it was much better than before treatment. Another epidural was performed, and four weeks following this he had dramatically improved, and had moved house without problems. This was now seven months after presentation. He has been fine since, ie for 16 months since the last epidural.

An MRI was obtained before his first epidural, which shows the prolapsed disc at L5/S1 (Figure 1). As it turned out this was not necessary for his management: it was obtained to save time in the event of surgery (discectomy), as I initially felt he would come to that. I could be criticised for this, although it did not use NHS money. 

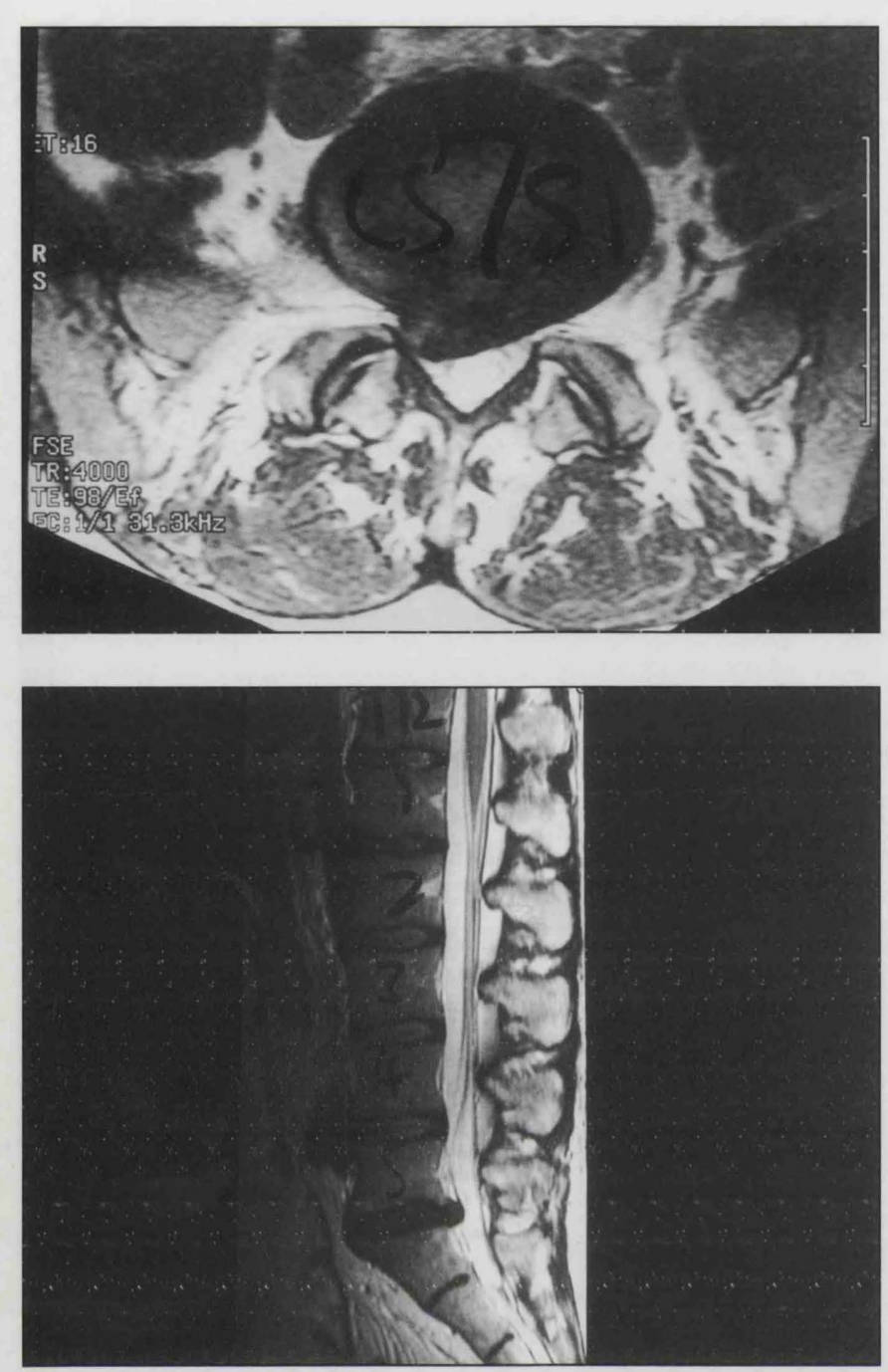

Figure I Sagittal and cross-section MRI views of the spine in Case I, showing a significant prolapse at the L5/S1 level on the right

\section{Case 2}

A 42-year-old man presented with a history of buttock and sacro-iliac region pain 18 months previously. He gave a history of hitting this region on a hard object when rising. At that stage it was documented that he had no restriction of SLR. He had had some physiotherapy treatment and NSAIDs, but with very little relief. The diagnosis at that time was 'sacro-iliac strain'. Several months later without any apparent cause he felt an increase in this pain, but now with pain radiating into his calf and ankle. There was marked restriction of SLR and he had a 'pelvic tilt'. A correct diagnosis of an acute disc problem was made, and he was advised to rest for a week. This did not help so an epidural was arranged which did not help much either. He had further osteopathic treatment which helped a little, and then further physiotherapy, and at just over a year from the original incident it was noted that his SLR was not restricted, but stretching his sacro-iliac joint caused his pain. He was booked for a sacro-iliac injection, but developed further sciatica while waiting: the sacro-iliac injection was performed, but with no immediate relief so at the same visit a further epidural was performed.

I saw him two months later (18 months after the initial incident), with sciatica, classical of the fifth lumbar root, with pain referred into the big toe and with 'gripping' of the ankle. Coughing and sneezing exacerbated his pain, and he was eased by lying on the other side to his pain.
His back was tilted at the lower lumbar level, away from the side of the pain (Figure 2). His back had restriction of movement, particularly flexion. He showed impairment of feeling on the outer border of the lower leg (L5 dermatome), and weakness of big toe dorsiflexion, (L5 again). His reflexes were normal as expected, and his SLR was 30 degrees on the affected side.

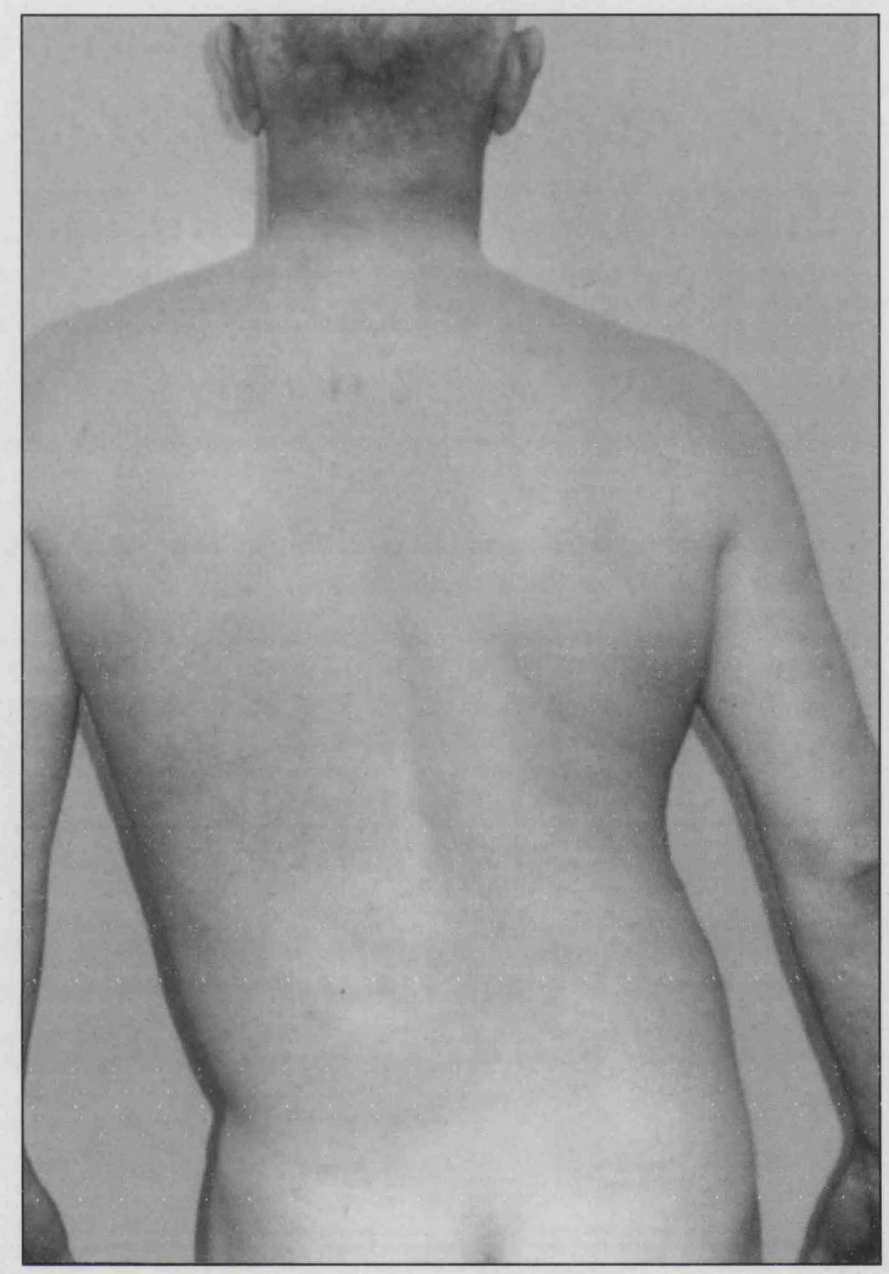

Figure 2 Case 2, with a spinal tilt away from the painful leg. Note that the pelvis is not tilted

A diagnosis of disc prolapse with L5 pressure was made, an MRI obtained (Figure 3), and the large L4/5 disc removed a month after he was seen. At the operation a large prolapse was found lateral to the nerve root, and another extruded fragment under the dura in the midline. He was in hospital for seven days, then rested at home for a month, before returning to a desk job.

After three and a half months he was starting a fire service physiotherapy course with a view to returning to active service at the end of the course. His back had straightened completely as shown in Figure 4.

\section{DISCUSSION}

There are several points illustrated nicely by these cases:

\section{Spinal tilt}

With a history of a disc prolapse, as outlined in paragraph 2 above, and as given with slight variations in both case histories, a spinal tilt is a sign of a disc prolapse. Figure 5 demonstrates why it occurs, which is, basically, to reduce the 

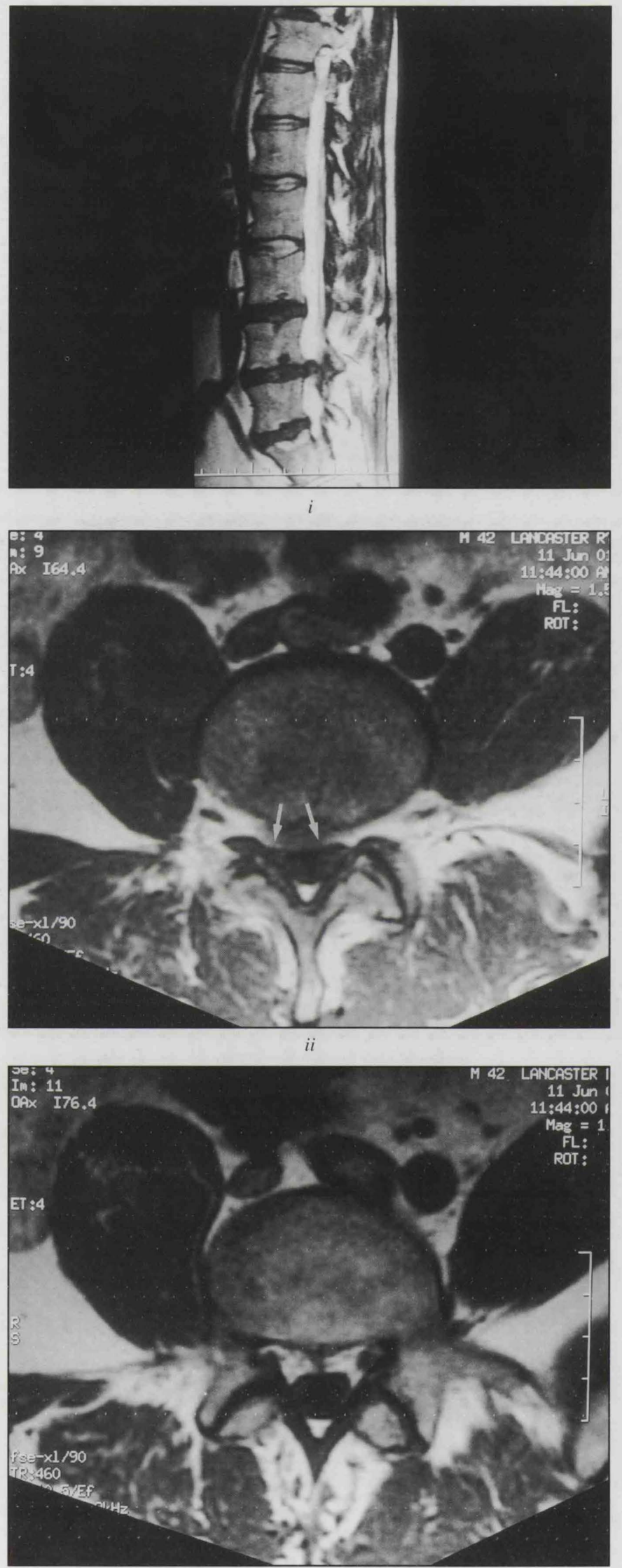

Figure 3 Sagittal and cross-section MRI views of the spine in Case 2, showing a large prolapse at LA/5 on the right side, with a cross-section of the normal spinal canal at L5/S1 for comparison.

stretch of the irritated nerve root. As the prolapse is usually lateral to the normal position of the root, the tilt is usually away from the side of the sciatica, as in both cases above, although note that the first one only showed the tilt in flexion.

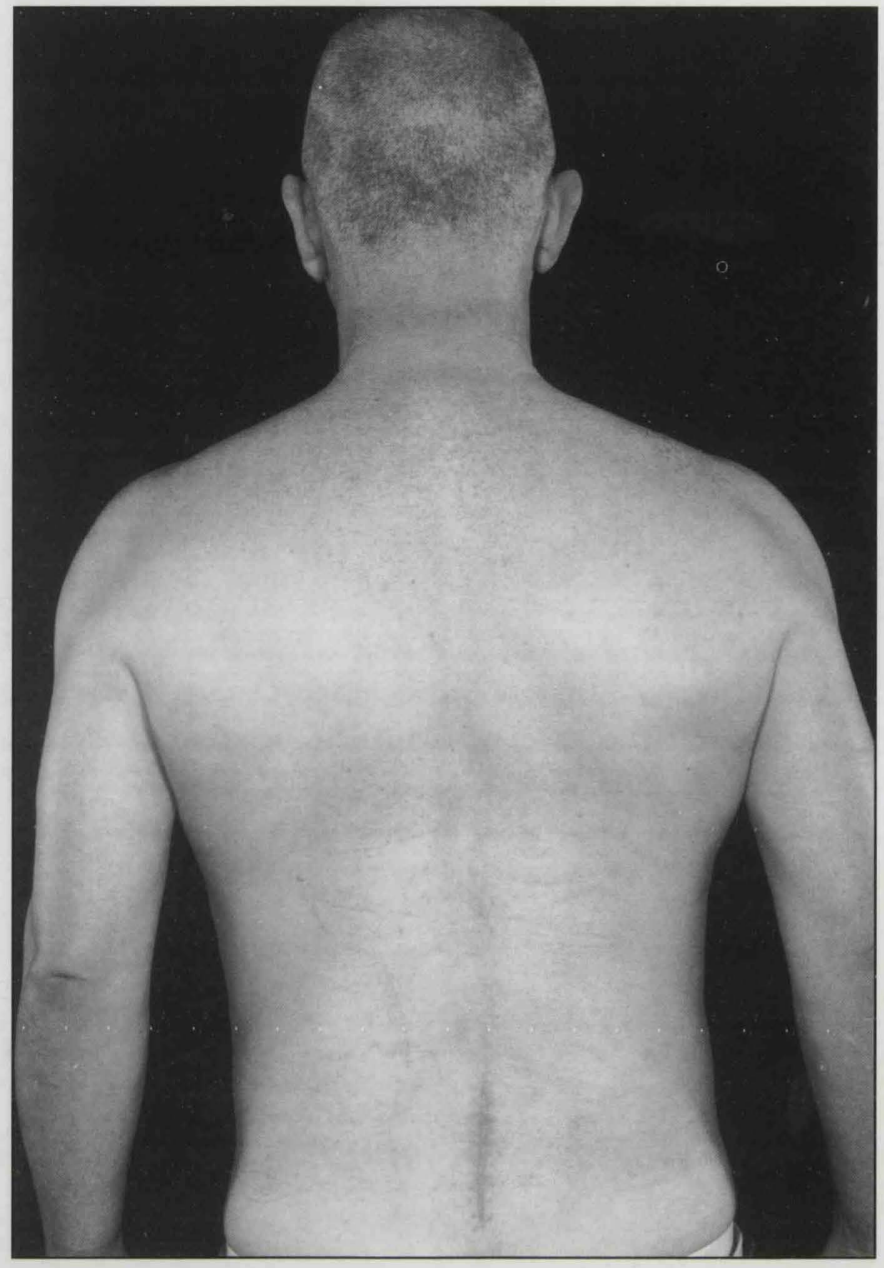

Figure 4 Case 2 after surgery. Spine now straight

Thus such a spinal tilt, with the usual history, is not caused by leg length discrepancy, pelvic maladjustment, sacro-iliac strain etc, and is NOT a pelvic tilt. A simple examination from behind the standing undressed patient confirms this.

\section{Sacro-iliac pain (SI pain)}

The second case presented with this, then developed the clinical picture of a disc prolapse with sciatica, then reverted back to merely having SI pain (when an injection was given), and then a disc again. I believe that the pain was referred all along from the responsible disc, and I believe that the vast majority patients with SI joint pain have it referred from the lower lumbar spine. Ankylosing spondylitis and other rheumatological diseases can cause SI joint pain, but there is no anatomical or physiological reason why a 'strain' of this joint should occur. This is not a long-held prejudice: I have made the diagnosis over the years, but subsequent treatment directed specifically towards the joint has never really been satisfactory. What happens, I think, is that the lumbar problem gradually improves, or it worsens, as in the second case.

\section{Epidural steroid injections}

There is massive prejudice from all sides against this treatment. I refer you to an evidence-based case report in the British Medical Journal ${ }^{(1)}$. The standard and ideal treatment for disc prolapses with sciatica that have not settled after rest for a few days or a week is outpatient physiotherapy, including traction. If it is not settling after several sessions the ideal treatment is an epidural. I would be happy if this 


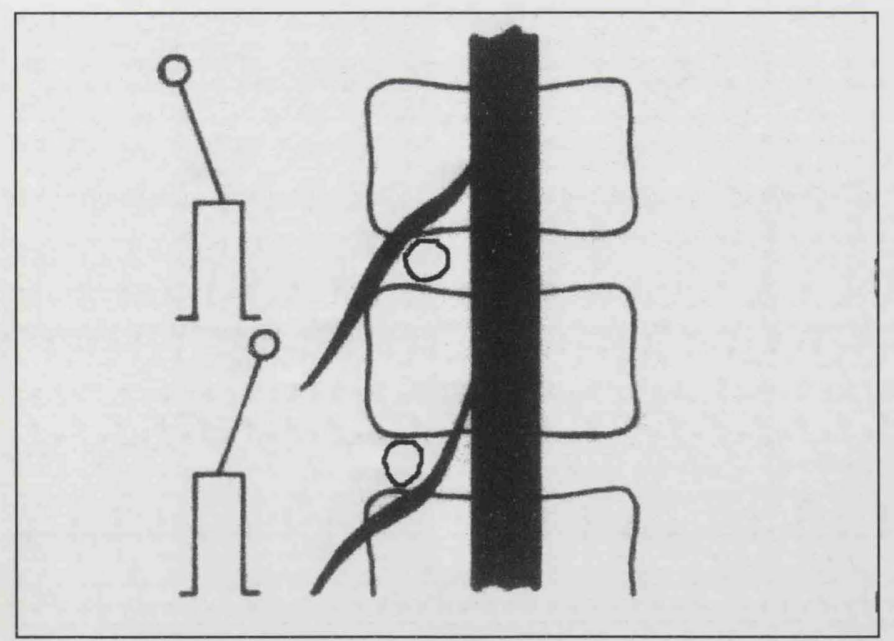

A

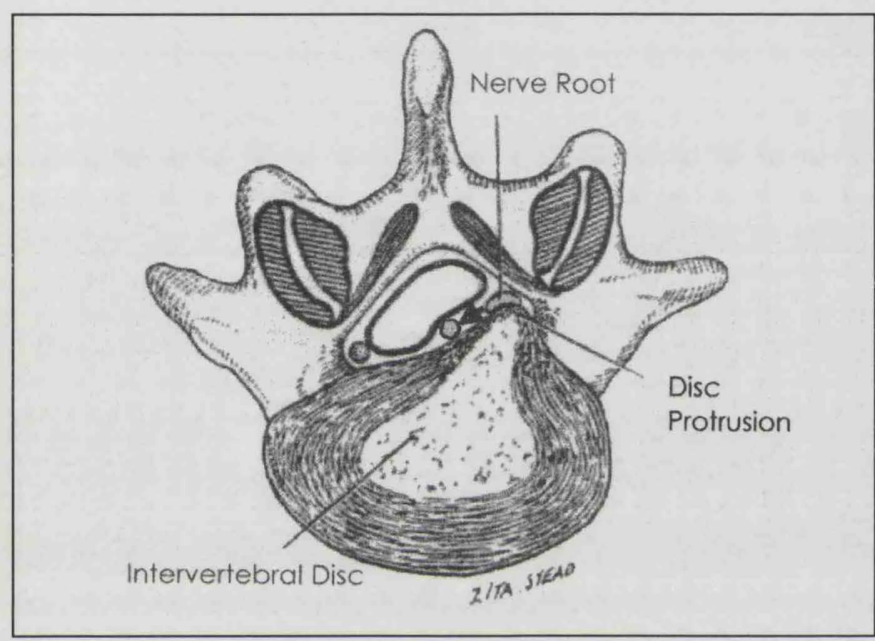

$B$

Figure 5 When a nerve root is stretched by a disc prolapse directly in front of it, the stretch can be relieved by movement of the spine to one side or the other as shown in A above, which causes the root to move down the slope of

the prolapse - medially as shown on the cross-section in B, which corresponds with the bottom situation, left in A above. It would move laterally in the top situation in A.

were to be in the local back pain guidelines, and open access for an epidural is a possibility. Certainly if I see such a patient I recommend both traction and an epidural (if the former has not been done, as in case 1), as neither treatment ensures full success. Case 1 illustrates the value of this standard treatment.

\section{Discectomy}

Discs causing serious motor impairment or central discs causing cauda equina (S234) pressure with bladder, bowel and sexual loss of function are obvious cases for surgical consideration, the latter as a matter of urgency, the former if not improving.

For all other cases, it is now well known that the results after five years are more or less the same whatever is done ${ }^{(2)}$. Clearly, therefore, a discectomy is not the first line of treatment. In the worst cases, however, like Case 2, it is worthwhile considering. It is the most rewarding operation I can do: the patient usually wakes up with a smile, as the sciatica has gone. Please note however, that success is conditional on there being a significant disc prolapse and on removal of the correct disc and all extruded fragments, if present.
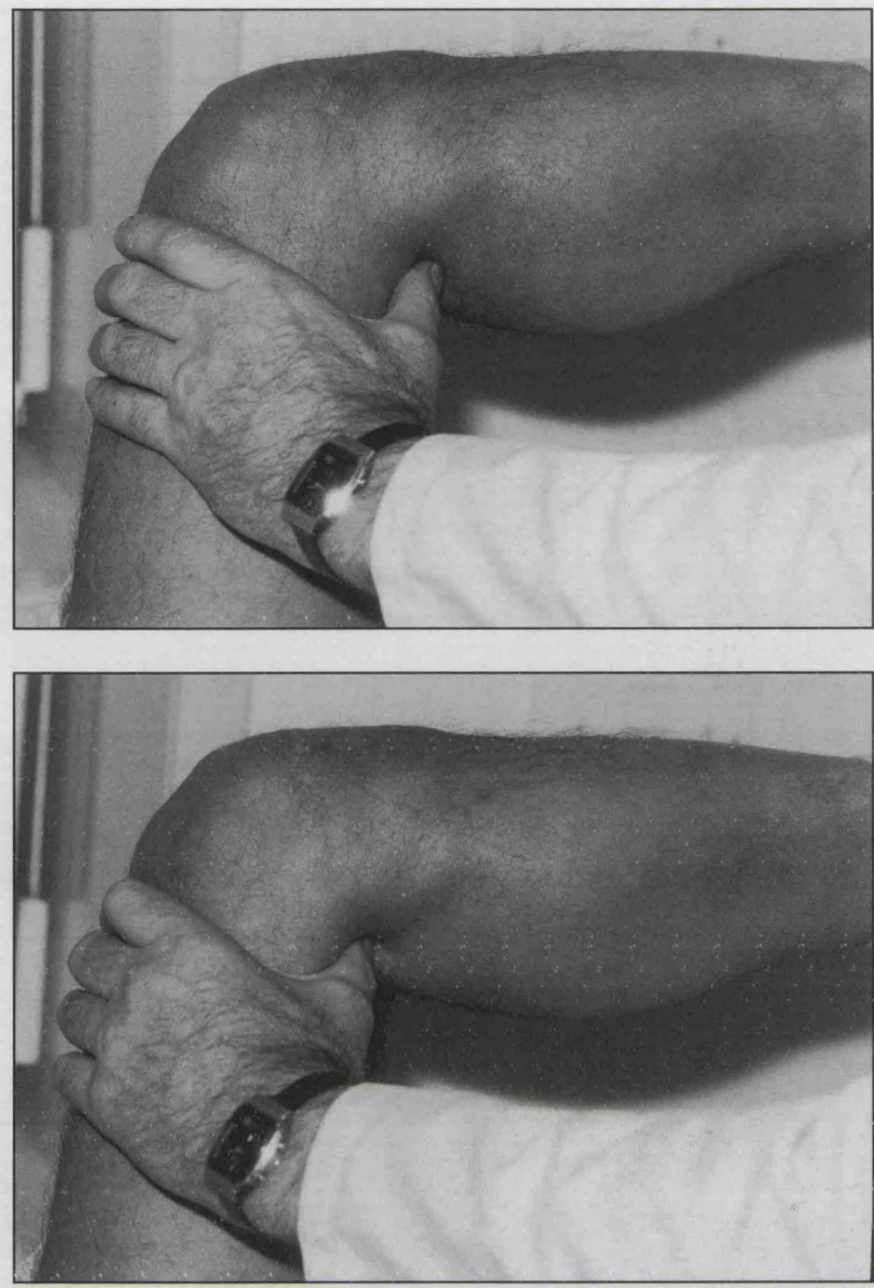

Figure 6 The bowstring test. Hip and knee flexed, thumb over the biceps tendon on the lateral popliteal fossa. Thumb compressing the biceps tendon in the lower picture, and with it the lateral popliteal nerve.

The first condition is decided clinically, ie the decision to consider an operation is entirely clinical: a significant disc prolapse is not difficult to diagnose, hopefully now by everybody, but the patients must have had a full course of physiotherapy with or without an epidural and not be improving.

The second condition, the correct level, is decided by an MRI, which reveals all. Thus an MRI should only be obtained after failure of conservative treatment.

The operation only removes the offending piece of 'crabmeat' from underneath the nerve root, and part of the remainder of the disc. It is got at by removing the ligamentum flavum between the laminae, and sometimes the edges of the laminae as well, but never in straight discectomies by removing the laminae and is thus not a destructive operation.

\section{Nerve root tension or irritation signs}

Straight leg raising (SLR). The most sensitive test is the bowstring test, mentioned in Case 1. This pulls the sciatic nerve roots in the spinal canal by deforming the lateral popliteal nerve as it is 'bowstrung' across the lateral popliteal fossa on 90 degree flexion of the knee. This is done by 'grabbing' the tibia just below the knee with your thumb in the popliteal fossa (Figure 6). The patient's leg and hence spine are stationary in this test, whereas with SLR, at a certain point, maybe 30-40 degrees, the lumbar spine moves 
and referred pain down the leg can occur. Positive tests reproduce the sciatica.

Case 2 illustrates that the SLR is a good indicator of 'external' disc activity ('internal' disc pathology is indicated by a discogenic history as given in paragraph 2 , minus sciatica). This may be mechanical, ie direct pressure, or merely irritation. Hence the patient started without restriction of SLR (I would say he had an internal disc problem), developed it with sciatica (external disc activity), improved with treatment, and finally had massive and consistent restriction.

It is my theory that the restriction of SLR is an indicator of inflammatory activity of the disc material.

This is very useful knowledge and explains the following:

a why most patients over 50 years of age with sciatica have no restriction of SLR. The cause is not discogenic at all, but due to narrowing of the lateral canals as a consequence of general spinal degeneration, discs and facet joints.

b why a small minority of patients with disc prolapses have little restriction of SLR. This happens when a fragment of disc has extruded into the spinal canal, which initially causes the classical picture, but which may settle down if the fragment moves slightly, and recur with violence if it moves back under a nerve root. Such a fragment will lose its nutrition and hence its inflammatory potential over a period of months, and so a time lapse from initial history to a clinical picture of sudden sciatica with no restriction of SLR can be due to a disc fragment.

c why recurrent disc prolapses after previous discectomy do not, in my experience, cause restriction of SLR with the sciatica, as the mainly fibrous material in the disc space is presumably low in inflammatory potential.

d why SLR is an indicator of whether an epidural will help the sciatica in the presence of a disc prolapse. That is, a patient with sciatica and discogenic history as in paragraph 2 , and restriction of SLR will usually be helped. Such a patient without much restriction is very unlikely to be helped.

Incidentally, it does not explain why an epidural helps the majority of patients with sciatica due to lateral spinal canal stenosis, who do not have restriction of SLR, but hey, I do not consider medicine to be an exact science!

6 Body position and activity (not particularly illustrated by the cases)

This may help to explain patients' progress or lack of it. In this context the aims of advice to the patient are to reduce pressure in the posterior (prolapsed) disc, and to prevent aggravation of irritation of the nerve root.
Reduction of pressure in the posterior part of a lumbar disc is achieved mostly by adopting a lordotic position ie an arched back bent backwards, and secondarily by taking weight off it: lying down, immersion in water or going into space!

Aggravation of nerve root irritation is achieved by not moving the leg, and not keeping it in a position in which it is pulled on, which is with hip flexion, especially with a straight knee. first:

Thus the following positions are graded for benefit, best

\begin{tabular}{|l|l|l|}
\hline position/activity & benefit - good & drawback - bad \\
\hline supine, hard surface & $\begin{array}{l}\text { lordosis induced } \\
\text { weight off }\end{array}$ & \\
\hline $\begin{array}{l}\text { sitting on high stool, } \\
\text { spine extended }\end{array}$ & $\begin{array}{l}\text { lordosis induced } \\
\text { hip extended }\end{array}$ & weight on \\
\hline standing & lordosis induced & weight on \\
\hline walking & $\begin{array}{l}\text { lordosis induced } \\
\text { (except up steep } \\
\text { slopes) }\end{array}$ & $\begin{array}{l}\text { weight on } \\
\text { leg movement }\end{array}$ \\
\hline $\begin{array}{l}\text { supine on soft surface } \\
\text { eg sagging bed }\end{array}$ & weight off & lordosis abolished \\
\hline $\begin{array}{l}\text { sitting in slouched } \\
\text { position eg driving }\end{array}$ & $\begin{array}{l}\text { lordosis abolished } \\
\text { (without lumbar roll) } \\
\text { weight on } \\
\text { hip flexed (maybe) }\end{array}$ \\
\hline
\end{tabular}

\section{Exceptions}

1 Some patients cannot lie supine, usually those with severe prolapses. My explanation for this is that the large prolapse or extruded disc fragment is effectively causing a local spinal stenosis. Canal cross-sectional area is decreased by lordosis and increased by forward flexion, hence stenotics can cycle better than walk. Such patients bend their knees when supine, or sit for relief.

2 Patients with chronic backs and acute prolapses cannot lie supine or prone for long, as the already symptomatic facet joints are further stretched.

Good luck!

\section{REFERENCES}

1 Samanta A, Beardsley J. Sciatica: which intervention? Evidence based case report. Br Med J 1999;319:302-3

2 Saal JA. Natural history and non-operative treatment of lumbar disc herniation. Spine 1996;21(suppl 42):2-9

\section{FURTHER READING}

Apley's System of Orthopaedics and Fractures. 7th ed pp. $367-71$ 